Research Article

\title{
Theoretical Study of the Effects of Carrier Transport, Capture, and Escape Processes on Solar Cells with Embedded Nanostructures
}

\author{
Chin-Yi Tsai \\ Department of Applied Physics, National University of Kaohsiung, Kaohsiung 811, Taiwan \\ Correspondence should be addressed to Chin-Yi Tsai; chinyitsai@hotmail.com
}

Received 12 January 2014; Accepted 18 January 2014; Published 24 February 2014

Academic Editor: Chien-Jung Huang

Copyright (C) 2014 Chin-Yi Tsai. This is an open access article distributed under the Creative Commons Attribution License, which permits unrestricted use, distribution, and reproduction in any medium, provided the original work is properly cited.

\begin{abstract}
A theoretical model is proposed to study the effects of carrier transport, escape, and capture processes on solar cells with embedded nanostructures. The theoretical results clearly indicate that the carrier transport, escape, and capture times are important physical quantities affecting the performance of solar cells with embedded nanostructures and they should be fully considered in device design, such as the selection of the optimal band-gap energy of the nanostructures. The beneficial results from the embedded nanostructures cannot be warranted. Slow escape processes and long transport time will make the nanostructures act as gigantic recombination sites and cause a detrimental effect on the bulk solar cell. The results show that solar cells embedded with nanostructures of very small band-gap energy materials will suffer from extremely slow escape processes due to a very large potential difference between the nanostructures and the bulk host material; therefore, their output photocurrent could be inferior to their bulk counterparts without the nanostructures. The beneficial results from the embedded nanostructures to the solar cells can only be realized by their long carrier lifetime and fast escape time.
\end{abstract}

\section{Introduction}

There exist ongoing efforts to improve the efficiency of solar cells. Solar cells embedded with nanostructures of smaller band-gap energy to form various quantum structures, such as quantum wells, wires, or dots, undoubtedly provide an interesting subject worth of more investigations $[1,2]$. The fact that the device performance demonstrated by quantumwell lasers is far more superior to their bulk counterparts $[3,4]$ somehow suggests that solar cells embedded with nanostructures might have better performances than their bulk counterpart. This is not a pure conjecture because the major advantage of embedding nanostructures of smaller band-gap energy into solar cells is that the nanostructures can absorb the additional photons below the band-gap energy of the bulk host material. In fact, using different materials to absorb different spectral portions of sunlight is a well-known practice for improving the efficiency of solar-cells. It can be achieved by using various methods and configurations, such as beam-splitting, mechanically stacking, heterojunction, and tandem structures. Embedding nanostructures of smaller band-gap energy directly into existing bulk solar cells certainly could achieve broader absorption of solar spectrum, as shown schematically in Figure 1. The nanostructures of smaller band-gap energy embedded in the bulk host material with larger band-gap energy, depending on their configurations and formations, will consequently constitute various quantum structures, such as quantum wells, wires, or dots. In fact, the quantum-well and quantum-dot solar cells have been experimentally realized and their performance is rather promising [1].

In spite of the promising features of solar cells embedded with nanostructures, there are some outstanding questions and problems in the device design remaining to be answered or solved, for example, the optimal band-gap energy of the nanostructures. As we know, deeper nanostructures can absorb a wider spectrum of the incident sunlight; however, they inevitably suffer from slower carrier escape processes. 


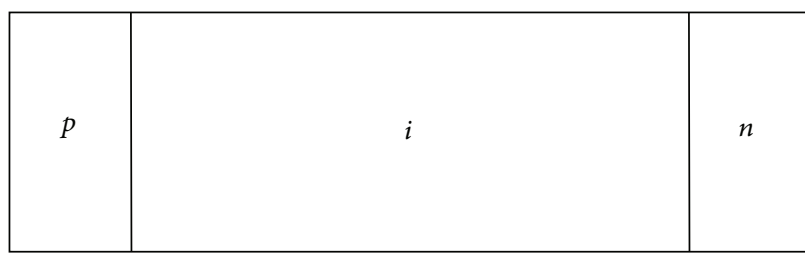

(a)

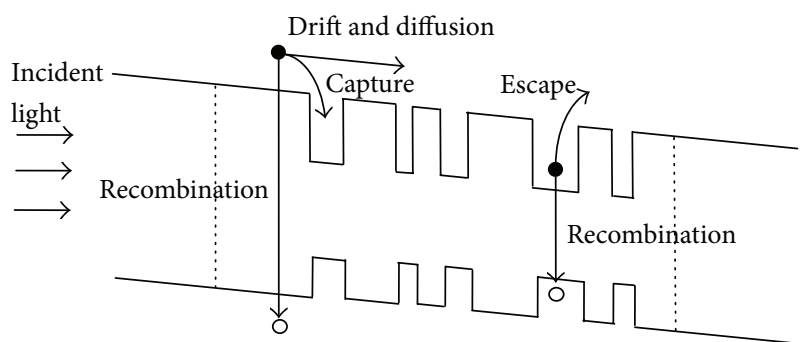

(c)

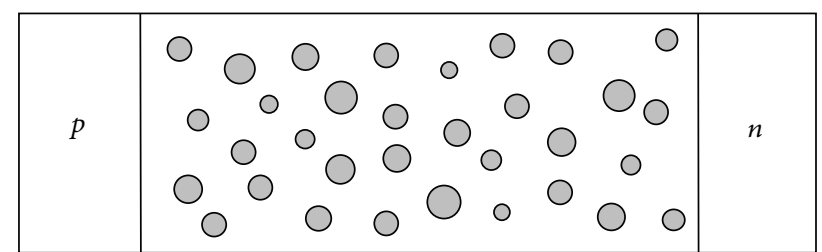

(b)

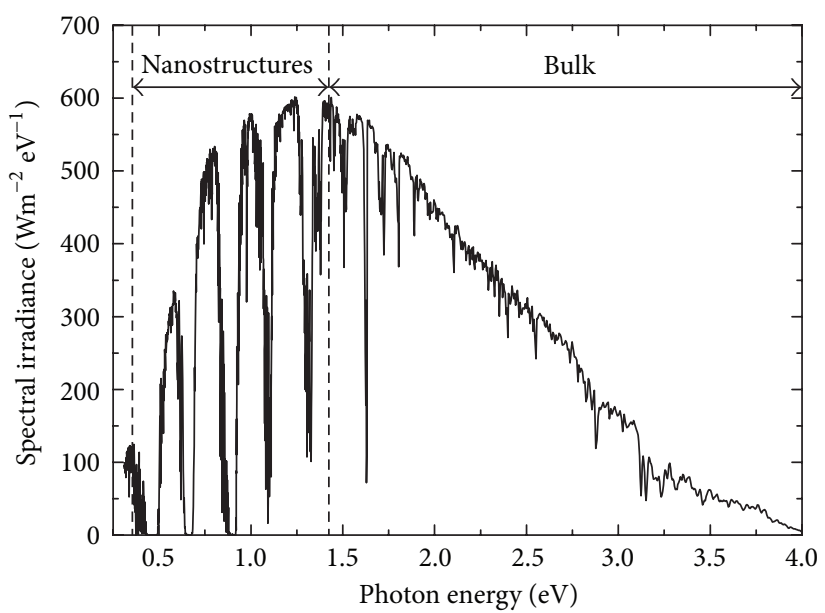

(d)

FIgURE 1: (a) A schematic diagram of a $p-i-n$ bulk solar cell. (b) A schematic diagram of a $p$-i- $n$ solar cell embedded with nanostructures in which the gray dots indicate the nanostructures. (c) A schematic energy band diagram of a $p-i-n$ solar cell embedded with nanostructures. (d) The ranges of light absorption from the bulk material (GaAs) and the nanostructures (InAs). The solar spectrum is AM1.5 normalized to the $1000 \mathrm{~W} / \mathrm{m}^{2}$ standard test condition.

In other words, carriers generated in the nanostructures with deep potential wells cannot efficiently escape from the nanostructures into the bulk materials and then transport out of the device to contribute as useful output photocurrents. In fact, carrier transport, capture, and escape processes are one of the key issues that determine the performance of quantum-well and quantum-dot lasers and their effects on the devices have been intensively investigated and discussed [3]. However, the effects of carrier transport, capture, and escape processes on solar cells embedded with nanostructures are not well studied yet [5-7].

\section{Theoretical Model}

In this work, a simple but rather illustrative theoretical model will be proposed to investigate the effects of carrier transport, capture, and escape processes on the photocurrent of solar cells embedded with nanostructures. A bulk $p-i-n$ solar cell is adopted and nanostructures (e.g., quantum dots) are formed in the bulk $i$-region, as shown schematically in Figure 1. To focus our discussion on the effects of carrier transport, capture, and escape processes on this solar cell, a simple $2-N$ model is used, in which the device is controlled by the carrier population of the unconfined states of the bulk material in the $i$-region, $N_{b}$, and that of the confined states of the nanostructures, $N_{w}$. Photocurrents contributed from the bulk $p$ - and $n$-regions are assumed to be omitted for simplicity. The assumptions and validity of this model have been discussed in our previous work for quantum-well lasers and will not be reiterated here [8]. Of course, more complicate models such as many- $N$ or distributed models with separated roles of electrons and holes can be implemented, but it is likely to make theoretical models less transparent and might obscure the essential results presented in this work. The rate equations of carrier populations $N_{b}$ and $N_{w}$ of this model are given as

$$
\begin{gathered}
\frac{d N_{b}}{d t}=\frac{I_{b}}{q}+\frac{N_{w}}{\tau_{\text {esc }}}-\frac{N_{b}}{\tau_{\text {cap }}}-\frac{N_{b}}{\tau_{b}}-\frac{N_{b}}{\tau_{d}}, \\
\frac{d N_{w}}{d t}=\frac{I_{w}}{q}-\frac{N_{w}}{\tau_{\text {esc }}}+\frac{N_{b}}{\tau_{\text {cap }}}-\frac{N_{w}}{\tau_{w}} .
\end{gathered}
$$

Here $q$ is the elementary charge. $I_{b}$ and $I_{w}$ are the photocurrents generated by the light absorption of the bulk material and the nanostructures; respectively. $\tau_{b}$ and $\tau_{w}$ are carrier lifetimes of the bulk material and the nanostructures, respectively. They are resulted from various recombination processes, such as, Shockley-Read-Hall (SRH), emission of photon, Auger, and interface recombination processes:

$$
I_{R}=I_{\mathrm{SRH}}+I_{\text {photon }}+I_{\text {Auger }}+I_{\text {interface }}+\cdots,
$$


where $I_{R b} / q=N_{b} / \tau_{b}$ and $I_{R w} / q=N_{w} / \tau_{w}$ represent the recombination currents in the bulk material and the nanostructures, respectively. The contributions of these recombination processes could be rather different for different materials and structures. For example, the SRH recombination is usually the dominant one in the indirect band-gap semiconductors, while the recombination via the emission of photon could be the most significant one in the direct bandgap semiconductors. On the other hand, for ill-form nanostructures, the interface recombination could be possibly the most dominant one. $\tau_{\text {esc }}$ and $\tau_{\text {cap }}$ are the carrier escape and capture times, respectively. $\tau_{d}$ is the carrier transport (i.e., drift and diffusion) time across the bulk $i$-region.

As shown schematically in Figure 1, (1) describes that carriers in the bulk material would recombine in the bulk material, captured into the nanostructures, or transport across the bulk region to contribute as the output photocurrent. Similarly, (2) describes that carriers in the nanostructures would either recombine inside the nanostructures or escape into the bulk materials. It should be noted that the carrier transport, capture, and escape models presented in (1) and (2) are rather universal and could be used for the cases of quantum wells, wires, dots, and other similar nanostructures by adopting their correspondent carrier lifetimes and carrier transport, capture, and escape times for specific cases which will be discussed in the following.

It has be theoretically shown that the carrier capture and escape times are in fact defined from the carrier net capture current $I_{\text {net }}$ as [8]

$$
\frac{I_{\text {net }}}{q}=\frac{N_{w}}{\tau_{\text {esc }}}-\frac{N_{b}}{\tau_{\text {cap }}} .
$$

If the carrier capture/escape processes proceed via the emission/absorption of phonons, it has been shown that the net capture current $I_{\text {net }}$ could be theoretically calculated from the first principle and thus the capture and escape times can be theoretically obtained for the case of quantum wells $[8,9]$. In our previous work, the carrier escape and capture processes between the unconfined bulk states and the confined quantum states have been calculated from the first principle by evaluating the carrier-polar optical phonon interactions, and the details of their physical meaning have been discussed and will not be repeated here for simplicity.

For solar cells, the devices effectively operate at DC steady state; that is, $d N_{b} / d t=d N_{w} / d t=0$; as a result, (1) and (2) can be presented in a form of the so called "charge-control model" as

$$
\begin{aligned}
& I_{b}-I_{\text {net }}-I_{R b}=I_{\lambda}, \\
& I_{w}+I_{\text {net }}-I_{R w}=0,
\end{aligned}
$$

where $I_{\lambda}$ are the output photocurrent, $I_{\text {net }}$ are net capture current, $I_{R b}$ and $I_{R w}$ are the recombination currents in the bulk and the nanostructures, respectively. Note that the output photocurrent is defined as $I_{\lambda} / q=N_{b} / \tau_{d}$. It describes that carriers in the bulk material must transport via drift and diffusion processes across the bulk $i$-region, before being recombined or captured into the nanostructures, and contribute as the useful output photocurrent. In fact, the carrier net capture current $I_{\text {net }}$, the recombination currents in the bull material $I_{R b}$, and the recombination current in the nanostructures $I_{R w}$ in (5) can be calculated from the first principles, and thus the correspondent time constants can be evaluated by $I_{\text {net }} / q=N_{b} / \tau_{\text {cap }}-N_{w} / \tau_{\text {esc }}, I_{R b} / q=N_{b} / \tau_{b}$, and $I_{R w} / q=N_{w} / \tau_{w}$ for a given specific operating condition.

From the addition of (3) and (4), the output photocurrent $I_{\lambda}$ is simply the superposition of the input photocurrent $I_{\text {photon }}=I_{b}+I_{w}$ generated by the absorption of the incident sunlight and the dark current $I_{\text {dark }}=I_{R b}+I_{R w}$ due to the recombination processes:

$$
I_{\lambda}=I_{\text {photon }}-I_{\text {dark }}=\left(I_{b}+I_{w}\right)-\left(I_{R b}+I_{R w}\right),
$$

as in the general case of a solar cell. The result of (6) clearly indicates that the superstition form of the photocurrent and the dark current for solar cells is still valid even with the inclusion of carrier transport, capture, and escape processes into the theoretical model. Similar forms of (6) have been used by several previous works without considering the effect of carrier escape and capture processes (i.e., $I_{\text {net }}=0$ ) in quantum-well solar cells [10].

There are several possible routes for a carrier to escape from a nanostructure to become a free carrier, such as phonon-assisted, photo-assisted, intraband and interband (i.e., Auger) carrier-assisted, and direct tunneling. Usually, phonon-assisted and direct tunneling are the two major channels for carrier escape processes. For typical $p-i-n$ solar cells with electric field in an $i$-region much less than $50 \mathrm{kV} / \mathrm{cm}$, the phonon-assisted escape process is known to be the dominant escape process in a quantum well [11]. In our previous work, the carrier escape and capture processes between the unconfined bulk states and the confined quantum states have been calculated from the first principle by evaluating the carrier-polar optical phonon interactions, and the details of their physical meaning have been discussed and will not be repeated here $[8,9]$. The results indicate that the quasi-Fermi energy of the confined states must be different from that of the unconfined states in order to have a net current flow between them. It should be noted that the escape and capture processes actually do not consume any photocurrent; they simply determine the proportion of the carrier populations (or more precisely, the quasi-Fermi energy levels) between the bulk material and the nanostructures. This quasi-Fermi energy separation between the bulk material and nanostructures will consequently modify the proportion of the dark recombination currents in the bulk and the nanostructures, and then the output current and voltage will be determined by the carriers in the bulk material transporting via drift and diffusion processes across the active region.

\section{Results and Discussion}

As it has been pointed out in our previous publication, the escape and capture times are not constants and they depend on the quasi-Fermi energy levels (and thus the carrier densities) in the bulk material and nanostructures. If this is the case, then the theoretical model of (1) and (2) should be 
solved and calculated numerically. However, if the escape and capture times are treated as time constants for a device at a given specific operating condition, the output photocurrent can be expressed by

$$
I_{\lambda}=\frac{I_{b}(\gamma+1)+I_{w} \gamma}{1+\alpha+\beta+\gamma+\gamma \beta},
$$

where the ratios between different time constants are defined as

$$
\alpha \equiv \frac{\tau_{d}}{\tau_{\text {cap }}}, \quad \beta \equiv \frac{\tau_{d}}{\tau_{b}}, \quad \gamma \equiv \frac{\tau_{w}}{\tau_{\text {esc }}} .
$$

These dimensionless parameters can be viewed as the indicators for the efficiencies of capture, transport, and escape processes, respectively. Note that if $1 / \tau_{b}=1 / \tau_{w}=0$ is assumed, a hypothetical case of infinite carrier lifetimes due to negligible recombination processes, the output photocurrent will become $I_{\lambda}=I_{b}+I_{w}$, which represents the theoretical maximum photocurrent that can be produced from a solar cell. In another hypothetical case of infinite carrier escape time, that is, $1 / \tau_{\text {esc }}=0$, the photocurrent will become

$$
I_{\lambda}=\frac{I_{b}}{1+\alpha+\beta}=I_{b} \frac{1 / \tau_{d}}{1 / \tau_{d}+1 / \tau_{b}+1 / \tau_{\text {cap }}} .
$$

It means that carriers cannot escape from the nanostructures and thus the nanostructures have no contribution for the output photocurrent at all, while the capture processes will make the nanostructures acting as recombination sites. Under such circumstances, solar cells embedded with nanostructures are even inferior to their bulk counterparts, and the inclusion of nanostructures will only degrade the efficiency of the device and totally lose its purpose. This just illustrates the importance of the carrier escape processes on the performance of solar cells embedded with nanostructures. Furthermore, if the carrier capture time is assumed to be infinite (i.e., $1 / \tau_{\text {cap }}=$ 0 ); in other words, the existence of the nanostructures will have no physical effects at all on the solar cell. In such cases, the output photocurrent can simply be calculated by

$$
I_{\lambda}=\frac{I_{b}}{1+\alpha}=I_{b} \frac{1 / \tau_{d}}{1 / \tau_{d}+1 / \tau_{b}} .
$$

Similar form as (10) has been proposed to study the effect of carrier transport on a bulk $p-i-n$ solar cell [12].

In our following discussion, $\tau_{d}$ is the combination of carrier drift and diffusion times:

$$
\frac{1}{\tau_{d}}=\frac{1}{\tau_{\text {drift }}}+\frac{1}{\tau_{\text {diff }}} .
$$

The drift time can be calculated by $\tau_{\text {drift }}=L_{b} / v_{\text {drift }}$, where $L_{b}$ is the width the active bulk $i$-region and $v_{\text {drift }}$ is the carrier average drift velocity, which is the sum of the contribution from electrons and holes $v_{\text {drift }}=v_{n}+v_{p}$ since the output photocurrent is contributed by electrons and holes separately. For a typical electric field $E \sim 10 \mathrm{kV} / \mathrm{cm}$ in a $p-i-n$ solar cell, the "high-field" effect in carriers' drift velocities might be

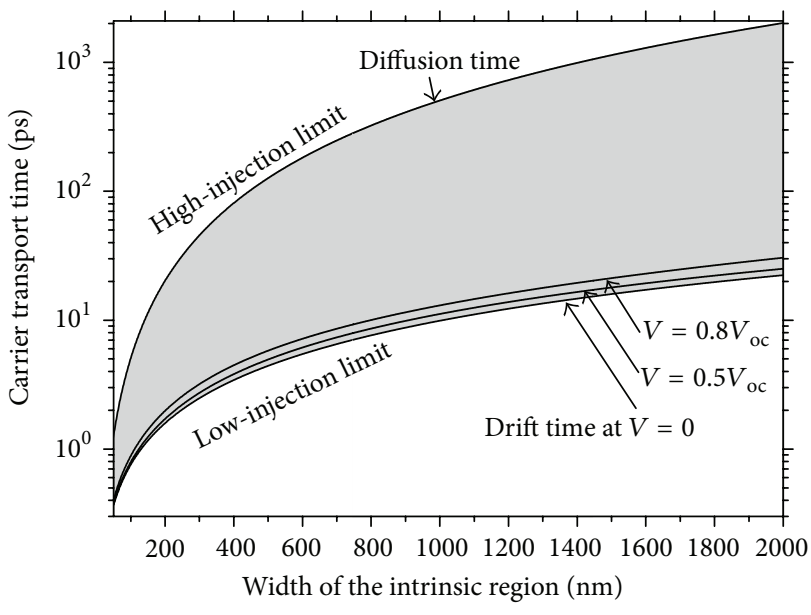

FIGURE 2: Carrier transport (diffusion and drift) times as a function of the width of the intrinsic region for different output voltages of a $p-i-n \mathrm{GaAs}$ bulk solar cell. $V_{\mathrm{oc}}$ stands for the open-circuit voltage. Note that the carrier transport is mainly limited by the drift process at low-injection conditions while it is limited by the diffusion process at high-injection conditions. The gray area indicates the possible range of carrier transport times.

considered. It has been suggested that carrier drift velocity can be calculated by

$$
v_{c}=\frac{\mu_{c} E}{\left[1+\left(\mu_{c} E / v_{\mathrm{sat}}\right)^{C}\right]^{1 / C}},
$$

where $C \approx 2$ for electrons and $C \approx 1$ for holes [13], $v_{\text {sat }}$ is the carrier saturation drift velocity, $\mu_{c}$ is the carrier mobility, and $E$ is the electric field in the active region. Although the actual electric field is a function of the position and is determined by the operating condition of the device, in the case of low injection, it could be roughly estimated by $E \approx$ $\left(V_{\mathrm{bi}}-V\right) / L_{b}$, where $V_{\mathrm{bi}}$ is the built-in voltage determined by the $n$ - and $p$-doping concentrations of the $n$ - and $p$-regions, respectively, and $V$ is the output voltage. The diffusion time can be calculated by $\tau_{\text {diff }}=L_{b}^{2} / 2 D$ where $D$ is the ambipolar carrier diffusion coefficient. If the electron and hole densities are assumed to be equal, then $2 / D=1 / D_{n}+1 / D_{p}$, which means that the ambipolar diffusion current is the sum of electrons and holes. Taking GaAs materials as an example, the calculated value of the drift time is $0.5 \sim 50$ ps while the diffusion time is $10 \sim 1000 \mathrm{ps}$ for the width of the $i$-region which varies from 0.1 to $2 \mu \mathrm{m}$, as shown in Figure 2. The diffusion current is usually rather small compared to the drift part, and it only becomes important at a very high-injection condition (e.g., solar cells operated with concentrators) where electron and hole densities are almost equal and the electric field resulted from the space charge in the $i$-region is very small and can be neglected. Under such circumstances, the slow carrier transport processes will certainly impede the output photocurrent of the solar cells, according to the result of (10). Therefore, the carrier transport processes are a vital factor that determines the performance of a solar cell. 


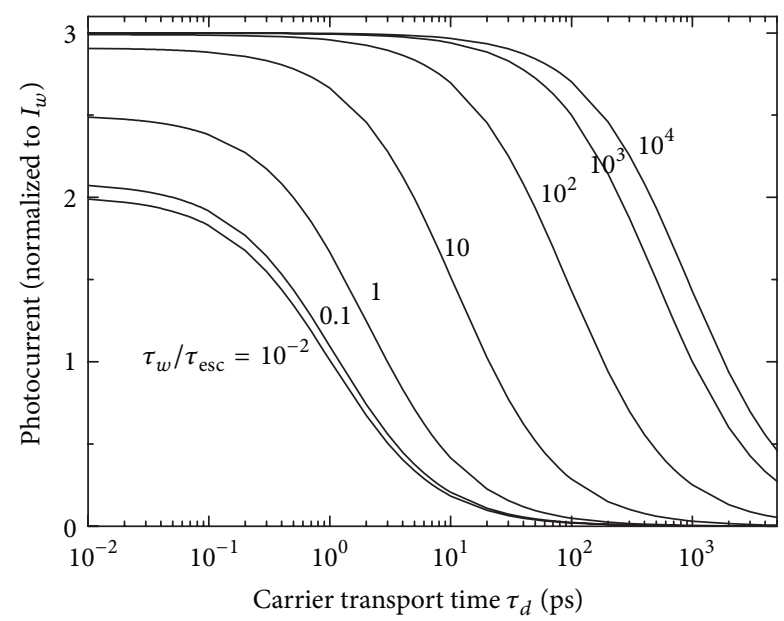

FIGURE 3: Total output photocurrent (normalized to the photocurrent contributed from nanostructures $I_{w}$ ) as a function of the carrier transport time $\tau_{d}$ at different ratios of carrier lifetime in nanostructures and the carrier escape time $\left(\tau_{w} / \tau_{\text {esc }}\right)$. Here, the carrier capture time is assumed to be $1 \mathrm{ps}$ and the carrier lifetime of the bulk material is $1 \mathrm{~ns}$.

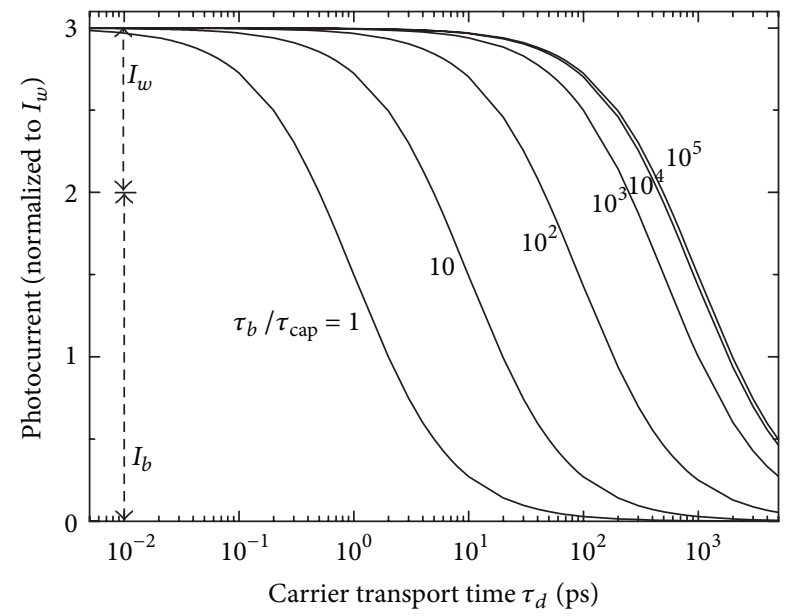

FIGURE 4: Total output photocurrent (normalized to the photocurrent contributed from nanostructures $I_{w}$ ) as a function of the carrier transport time $\tau_{d}$ at different ratios of carrier lifetime in the bulk material and the carrier capture time $\left(\tau_{b} / \tau_{\text {cap }}\right)$. Note that the dot line indicates the photocurrent contributed from the bulk host material $I_{b}$ and the nanostructures $I_{w}$ at very small carrier transport time. Here, the carrier escape time is assumed to be 1 ps and the carrier lifetime of nanostructures is $1 \mathrm{~ns}$.

Based on the above theoretical model, the calculated results of carrier transport time on the output photocurrent of a solar cell embedded with nanostructure are shown in Figures 3 and 4. Usually the carrier lifetime for direct bandgap materials, such as GaAs, is in the range of nanoseconds if the spontaneous emission of photons is the dominant recombination process. The escape time for nanostructures with small potential difference from the bulk material via the absorption of a single phonon is typically in the range of picoseconds for carrier-polar optical phonon interactions.

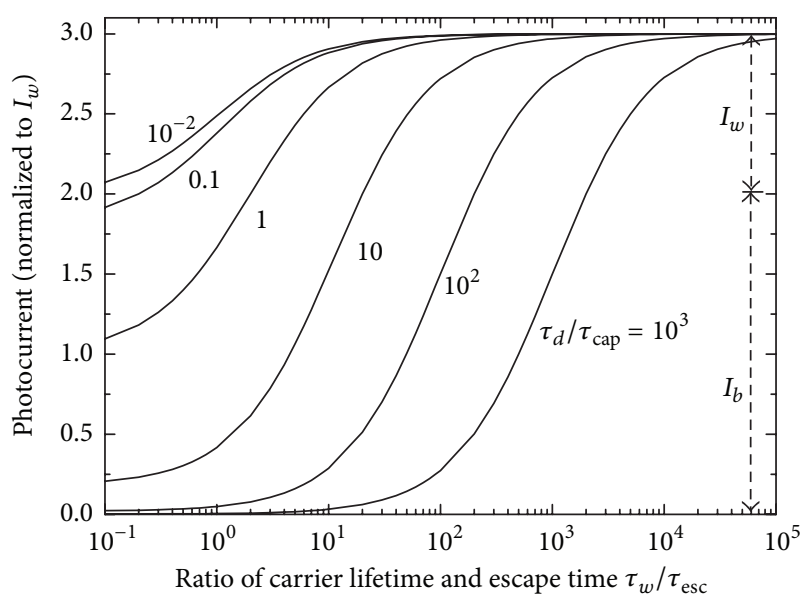

FIGURE 5: Total output photocurrent (normalized to the photocurrent contributed from nanostructures) as a function of the ratio carrier lifetime in nanostructures and the carrier escape time $\left(\tau_{w} / \tau_{\text {esc }}\right)$ at different ratios of the carrier transport time and the carrier capture time $\left(\tau_{d} / \tau_{\text {cap }}\right)$. Note that the dot line indicates the maximal photocurrent contributed from the bulk material $I_{b}$ and the nanostructures $I_{w}$ with the minimal effects of carrier escape and capture processes. Note that the carrier lifetime of the bulk materials is assumed to be $1 \mathrm{~ns}$.

As a result, $\gamma \approx 10^{3}$ can be reasonably assumed for such a case and the carrier transport processes will have a negligible effect on the output photocurrent if the carrier transport time is smaller than $10 \mathrm{ps}$, as shown in the results of Figure 3. On the other hand, for nanostructures with very large potential difference from the bulk material, carriers can only escape from the nanostructures by absorbing several phonons and the escape time could be in the range of nanoseconds. If $\gamma \approx 1$ is assumed for such a case, the nanostructures could possibly contribute the output photocurrent only if carrier transport time is smaller than $1 \mathrm{ps}$, as shown in the results of Figure 3.

The effect of the carrier escape processes is a vital factor determining the usefulness of the nanostructures in the solar cell. This can be fully appreciated from the calculated results in Figures 5 and 6 . For carriers suffering from an extremely large escape time and thus a large ratio of carrier lifetime and escape time, if carriers have very slow transport process as well, the device can only generate a negligible amount of output photocurrent. Under such circumstances, the nanostructures in fact act as gigantic recombination sites and have no beneficial effect at all on the bare bulk solar cell.

In fact, the effects of carrier transport, capture, and escape processes are not separated incidents working independently from each other. On the contrary, they are closely intertwined with each other and affect the performance of solar cells simultaneously. The overall effect of carrier transport, capture, and escape processes on the output photocurrent can be illustrated from the calculated results of Figure 7. It clearly demonstrates that the beneficial results from the embedded nanostructures cannot be warranted. Slow escape processes and long transport time will turn the nanostructures into gigantic recombination sites and cause a detrimental effect on the bulk solar cell, rather than a beneficial one. 


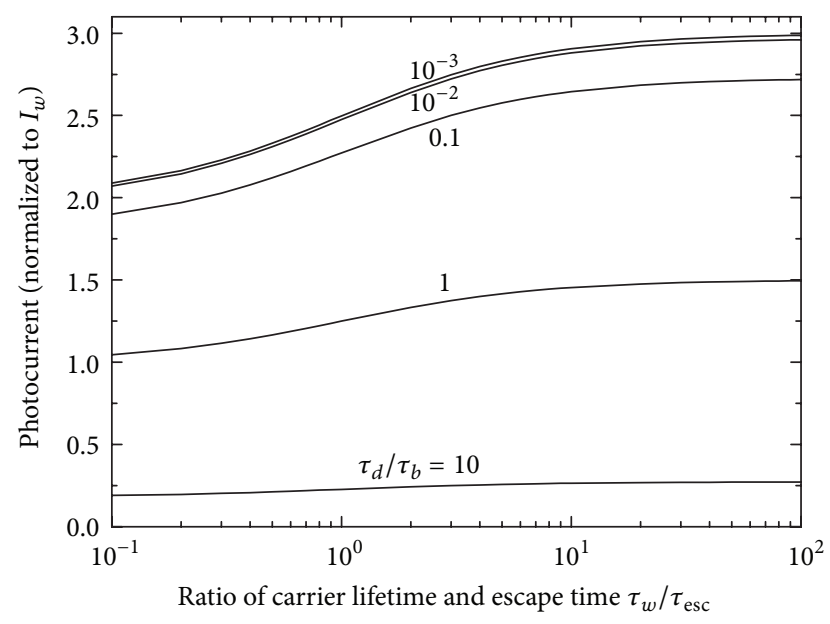

Figure 6: Total output photocurrent (normalized to the photocurrent contributed from nanostructures) as a function of the ratio carrier lifetime in nanostructures and the carrier escape time $\left(\tau_{w} / \tau_{\text {esc }}\right)$ at different ratios of the carrier transport time and the carrier lifetime of the bulk material $\left(\tau_{d} / \tau_{b}\right)$. Note that the carrier capture time is assumed to be $1 \mathrm{ps}$.

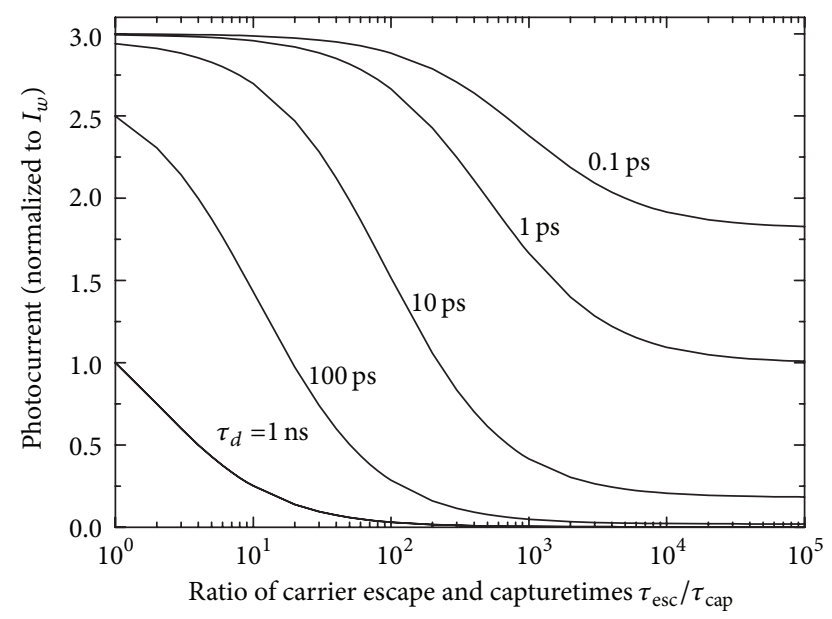

FIGURE 7: Total output photocurrent (normalized to the photocurrent contributed from nanostructures) as a function of the carrier capture/escape time ratio $\left(\tau_{\text {esc }} / \tau_{\text {cap }}\right)$ at different carrier transport times. Here the carrier lifetimes in the nanostructures and the bulk material are assumed to be $1 \mathrm{~ns}$.

The device design issue regarding the choice the nanostructure material with the optimal band-gap energy can be calculated and discussed by using the theoretical model presented in this work. The carrier capture/escape processes mainly proceed via the emission/absorption of phonons with limited amount of energies and thus strongly depend on the band-edge energy difference between the nanostructures and the bulk material. It has been shown that the carrier capture/escape time ratio almost exponentially depends on the potential difference between the band edges of the quantum wells and the bulk barriers as

$$
\frac{\tau_{\text {cap }}}{\tau_{\text {esc }}} \propto \exp \left(-\frac{\Delta E}{k_{B} T}\right)
$$

where $k_{B}$ is the Boltzmann constant, $T$ is the carrier temperature, and $\Delta E$ is the band-edge energy differences of the conduction bands for electrons or the valence bands for holes $[8,9]$. However, carriers capture/escape processes in other nanostructures, such as quantum dots, might be rather different from the cases of quantum wells, but the general relation given by (13) could be still observed, as long as the carriers proceed their capture/escape processes via the emission/absorption of phonons.

The output photocurrents as a function of the bandgap energies of the nanostructure materials are calculated and the results are shown in Figure 8 with different carrier transport/capture time ratios. The calculated results clearly demonstrate that there exists an optimal band-gap energy of the nanostructures for achieving the maximal output photocurrent. Nevertheless, the exact value of the optimal band-gap energy critically depends on the carrier lifetime in the nanostructures.

In the cases of very fast carrier transport, the results shown in Figure 8(a) indicate that the nanostructures generally have beneficial effect on the output photocurrent. On the contrary, in the cases of very slow carrier transport, as shown in Figure 8(c), the photocurrent contributed from the nanostructures becomes less significant, while the photocurrent contributed from the bulk material is severely degraded by the carrier escape processes. Nanostructures with very deep potential depth could have detrimental effect on the bare bulk solar cell even in the cases of very long carrier lifetime (i.e., nearly perfect material conditions). It should be noted that, if a solar cell embedded with nanostructures designed to be operated under light concentration, the devices will then operate at a high-injection condition and thus with a longer transport time. As a result, the effects of carrier transport, capture, and escape processes will become more important and should therefore be considered in the design for such devices operated with solar concentrators.

\section{Conclusion}

In conclusion, a theoretical model is proposed to study the effects of carrier transport, capture, and escape processes on solar cells with embedded nanostructures. The calculated results show that nanostructures with very small band-gap energies will suffer from extremely slow escape processes. Under such circumstances, carriers cannot efficiently escape from the nanostructures and thus the nanostructures have a negligible contribution for the output photocurrent, while the capture processes will make the nanostructures act as recombination sites. If this is the case, solar cells embedded with nanostructures are even inferior to their bare bulk counterparts, and the inclusion of nanostructures could degrade the performance of the device and totally loses its purpose.

The optimal band-gap energies of the nanostructure materials for achieving the maximum photocurrent are calculated and discussed by using the theoretical model given in this work. The results clearly demonstrate that the effects of carrier transport, capture, and escape processes on a solar cells embedded with nanostructures are very significant and 


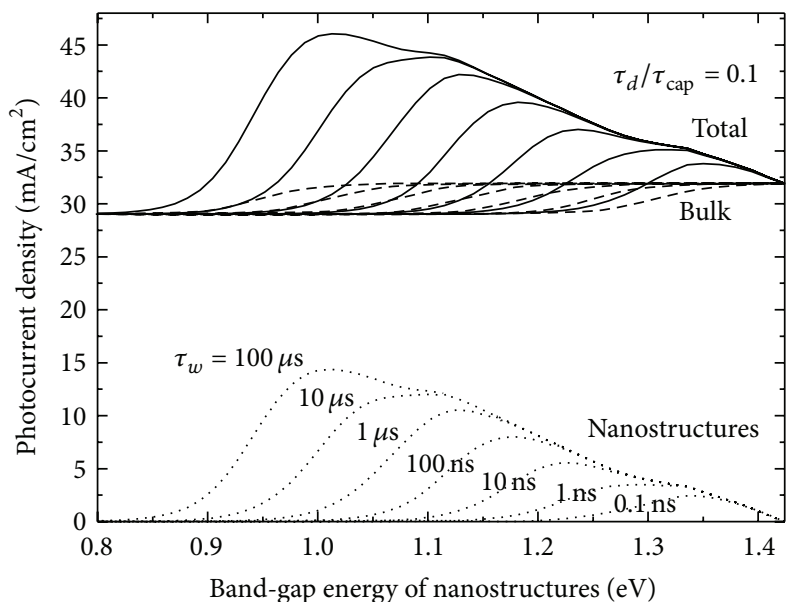

(a)

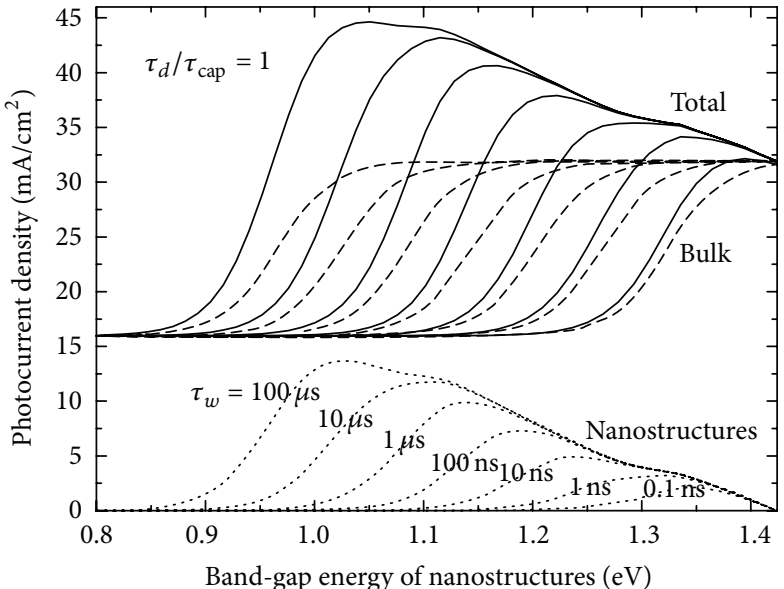

(b)

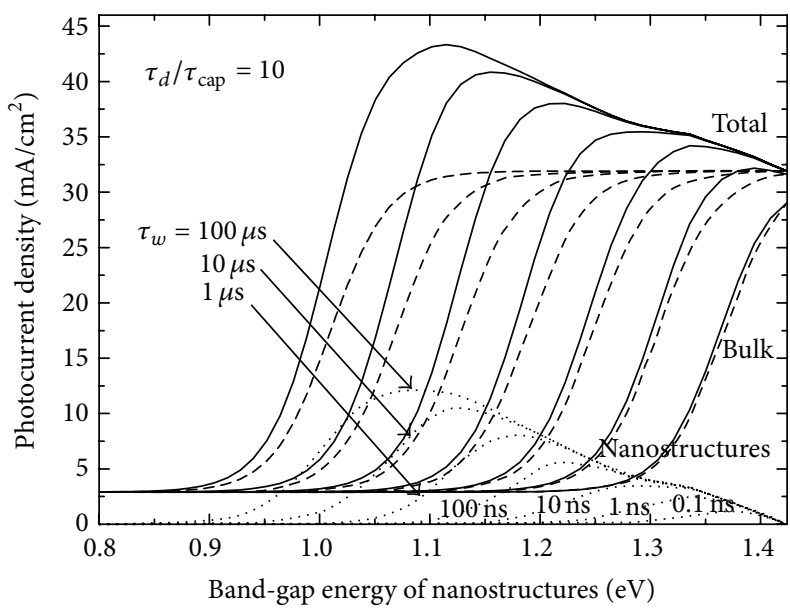

(c)

FIGURE 8: The output photocurrent density contributed from the nanostructures (dot line), the bulk material (dash line), and their sum (solid line) as a function of the band-gap energy of the nanostructures at different carrier lifetimes of the nanostructures $\tau_{w}$. (a) The ratio of the carrier transport time and the carrier capture time is assumed to be 0.1 to represent the ideal cases of very fast carrier transport processes in the bulk $i$-region. (b) The ratio of the carrier transport time and the carrier capture time is assumed to be 1. (c) The ratio of the carrier transport time and the carrier capture time is assumed to be 10 to represent the cases of very slow carrier transport processes in the bulk $i$-region.

should be considered in the device design, especially in designing devices operated with solar concentrators.

\section{Conflict of Interests}

The author declares that there is no conflict of interests regarding the publication of this paper.

\section{Acknowledgment}

This work is partially funded by the Bureau of Energy, Ministry of Economic Affairs, Taiwan.

\section{References}

[1] M. Mazzer, K. W. J. Barnham, I. M. Ballard et al., "Progress in quantum well solar cells," Thin Solid Films, vol. 511-512, pp. 7683, 2006.
[2] A. J. Nozik, "Quantum dot solar cells," Physica E, vol. 14, no. 1-2, pp. 115-120, 2002.

[3] P. S. Zory Jr., Quantum Well Lasers, Academic Press, San Diego, Calif, USA, 1993.

[4] V. M. Ustinov, Quantum Dot Lasers, Oxford University Press, Oxford, UK, 2003.

[5] A. Zachariou, J. Bames, K. W. J. Bamham et al., "A carrier escape study from InP/InGaAs single quantum well solar cells," Journal of Applied Physics, vol. 83, no. 2, pp. 877-881, 1998.

[6] S. M. Ramey and R. Khoie, "Modeling of multiple-quantumwell solar cells including capture, escape, and recombination of photoexcited carriers in quantum wells," IEEE Transactions on Electron Devices, vol. 50, no. 5, pp. 1179-1188, 2003.

[7] A. Alemu, J. A. H. Coaquira, and A. Freundlich, "Dependence of device performance on carrier escape sequence in multiquantum-well p-i-n solar cells," Journal of Applied Physics, vol. 99, no. 8, Article ID 084506, 5 pages, 2006. 
[8] C. Y. Tsai, C. Y. Tsai, Y. H. Lo, R. M. Spencer, and L. F. Eastman, "Nonlinear gain coefficients in semiconductor quantum-well lasers: effects of carrier diffusion, capture, and escape," IEEE Journal on Selected Topics in Quantum Electronics, vol. 1, no. 2, pp. 316-330, 1995.

[9] C. Y. Tsai, L. F. Eastman, Y. H. Lo, and C. Tsai, "Breakdown of thermionic emission theory for quantum wells," Applied Physics Letters, vol. 65, no. 4, pp. 469-471, 1994.

[10] N. G. Anderson, "Ideal theory of quantum well solar cells," Journal of Applied Physics, vol. 78, no. 3, pp. 1850-1861, 1995.

[11] D. M. T. Kuo and Y. C. Chang, "Dynamic behavior of electron tunneling and dark current in quantum-well systems under an electric field," Physical Review B, vol. 60, no. 23, pp. 15957-15964, 1999.

[12] J. Nelson, The Physics of Solar Cells, Imperial College Press, London, UK, 2003.

[13] S. M. Sze and K. K. Ng, Physics of Semiconductor Devices, John Wiley \& Sons, New York, NY, USA, 3rd edition, 2007. 

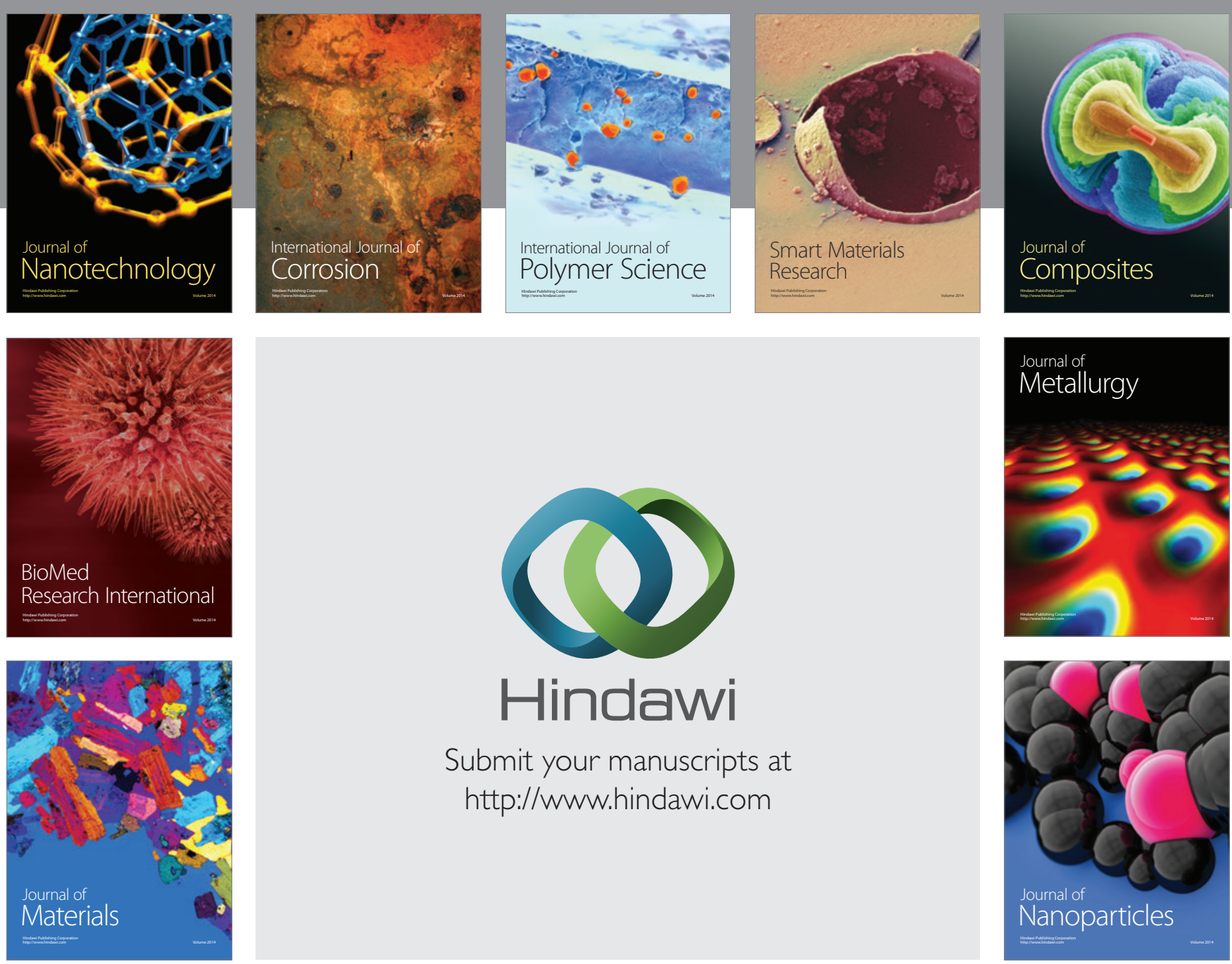

Submit your manuscripts at http://www.hindawi.com
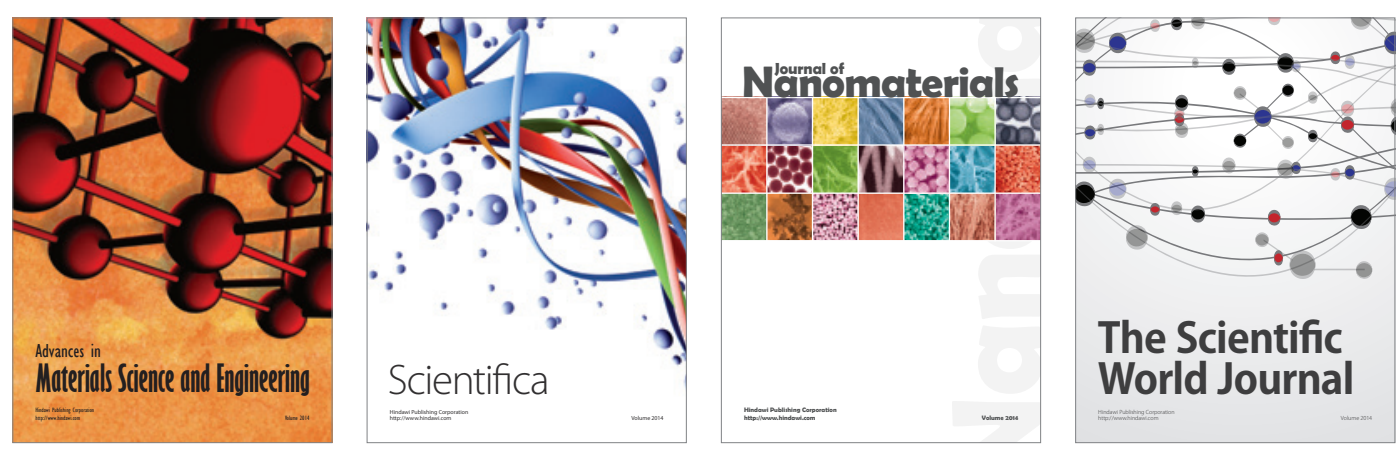

\section{The Scientific World Journal}
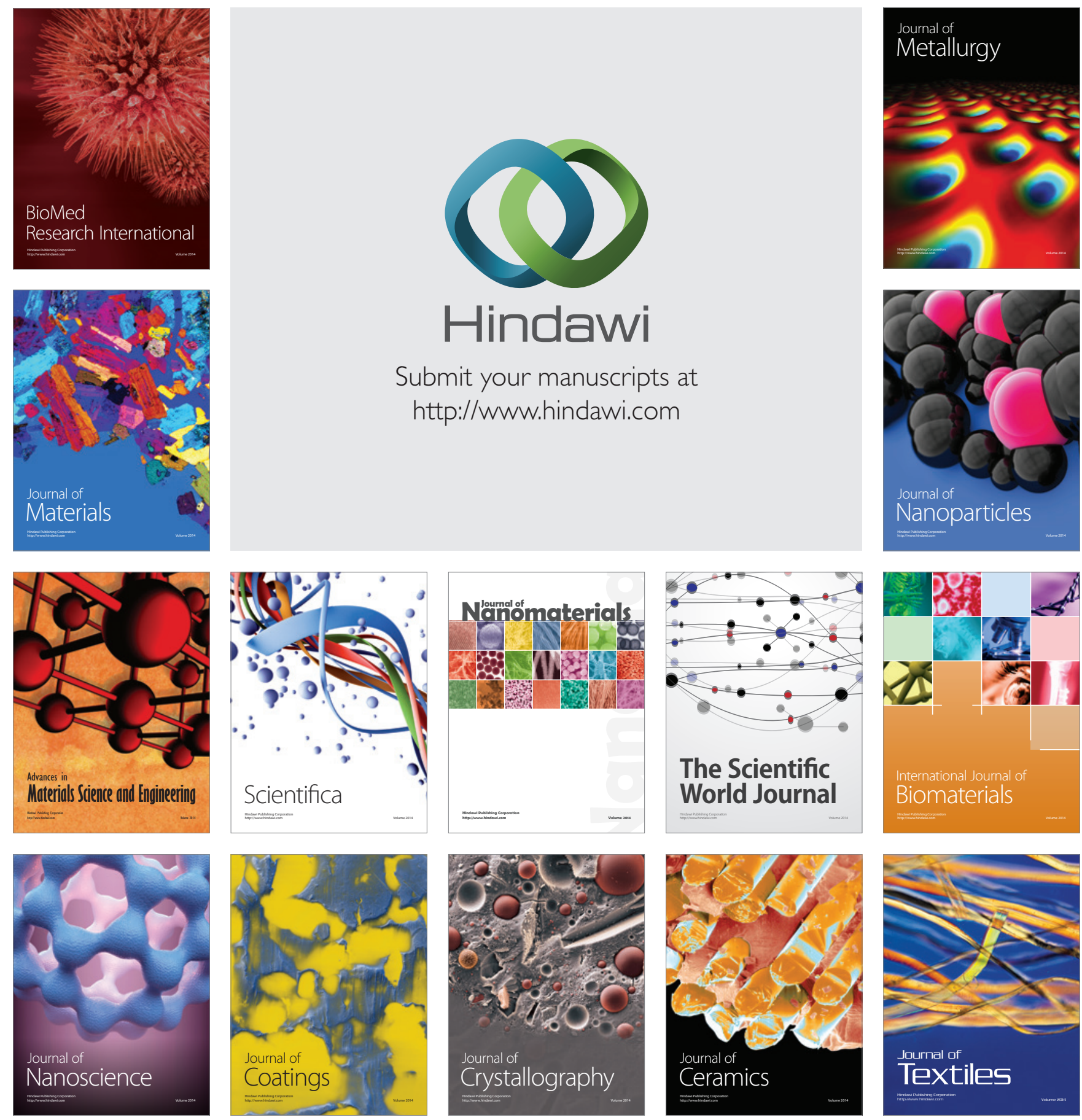